Bull. Mater. Sci., Vol. 2, Number 3, August 1980, pp. 207-216. (C) Printed in India.

\title{
Positron annihilation studies of rare-earth mixed valence compounds
}

\author{
K P GOPINATHAN, C S SUNDAR, B VISWANATHAN and \\ A BHARATHI \\ Reactor Research Centre, Kalpakkam 603 102, India
}

MS received 16 April 1980

\begin{abstract}
Positron lifetime has been measured in SmS under external pressures from 0 to $\sim 16 \mathrm{kbar}$, and in $\left(\mathrm{Sm}_{\mathbf{0} .8} \mathrm{Gd}_{0.2}\right) \mathrm{S}$ at temperatures from $10 \mathrm{~K}$ to $300 \mathrm{~K}$. An abrupt drop in the lifetime was observed at the valence transition at a pressure of $6.5 \mathrm{kbar}$ in $\mathrm{SmS}$ and at a temperature of $\sim 120 \mathrm{~K}$ in $\left(\mathrm{Sm}_{0.8} \mathrm{Gd}_{0.2}\right) \mathrm{S}$. Measurement of the Doppler broadening of the annihilation photons was made in $\left(\mathrm{Sm}_{0.3} \mathrm{Gd}_{\mathbf{0 . 2}}\right) \mathrm{S}$ as a function of temperature. The line shape parameter of the Doppler broadened spectrum also showed an abrupt change at the transition. Angular correlation of annihilation photons was measured in the semiconducting phase of SmS and in the metallic phase of $\left(\mathrm{Sm}_{0.8} \mathrm{~Tb}_{0.2}\right) \mathrm{S}$. The correlation profile in the mixed valence (metallic) phase was broader than that in the semi-conducting phase. These results have been explained on the basis of the electronic structure of the semiconducting and the metallic phases of these materials.
\end{abstract}

Keywords. Positron annihilation ; lifetime ; angular correlation ; Doppler broadening; mixed valence ; rare-earths ; phase transition.

\section{Introduction}

The rare-earth ions in the monochalcogenides (sulphides, selenides and tellurides) of $\mathrm{Sm}, \mathrm{Eu}$ and $\mathrm{Yb}$ and EuO and $\mathrm{TmTe}$ are divalent in character. This has been observed from a comparison of the measured lattice parameters of these compounds with those of the neighbouring trivalent rare-earth chalcogenides. These compounds undergo an electronic phase transition on application of high pressures (Jayaraman et al 1970, 1975a, b). The transitions are isostructural $(\mathrm{NaCl} \rightarrow \mathrm{NaCl}$ structure). In some compounds like $\mathrm{SmS}$ the transition is discontinuous while in some others like SmSe and SmTe the transition is continuous. The divalent compounds are semiconductors. On application of high pressures they undergo a transition to the metallic state. In the case of $\mathrm{SmS}$ the lattice parameter changes from $5.97 \AA$ to $5 \cdot 73 \AA$ as a result of the transition. The lattice parameter for the high pressure phase of SmS is higher than the interpolated value from trivalent rare-earth sulphides. The valence of $\mathrm{Sm}$ in the high pressure phase is 2.8 as estimated from the lattice parameter. Solid solutions of $\left(\mathrm{Sm}_{1-»} \mathrm{Gd}_{\star}\right) \mathrm{S},\left(\mathrm{Sm}_{1-\infty} \mathrm{Tb}_{\star}\right) \mathrm{S}$ and $\left(\mathrm{Sm}_{1-1} \mathrm{Y}_{e}\right) \mathrm{S}$ show a metallic lustre for the range of values of $x$ between $\sim 0.15$ 
and $\sim 0.28$. The lattice parameter also changes discontinuously to $5.73 \AA$, similar to the high pressure phase of SmS. When these compounds are cooled to low temperatures they change to a black powder explosively, turning into nonmetallic state (Jayaraman et al 1973). Mössbauer isomer shifts and x-ray photoelectron spectra of the semiconducting and metallic phases of SmS also indicate the mixed valent nature of the metallic phase (Coey et al 1976; Campagna et al 1974; Pollak et al 1974).

The electronic structure of SmS has been studied in both the phases by optical spectroscopy (Batlogg et al 1976; Suryanarayanan 1978). In the semiconducting phase of SmS, the Sm has the electronic configuration $4 \mathrm{f}^{6} 5 \mathrm{~d}^{0}$. The transition to the metallic phase involves the delocalisation of a $4 f$ electron into the $5 d$ band thereby changing the configuration to $4 f^{5} 5 d^{1}$. In the semiconducting phase of $\mathrm{SmS}$, the conduction bands derived from $5 d-6 s$ states of $\mathrm{Sm}$ are separated by an energy gap from the valence band derived from $p$ states of sulphur (see figure 1). The atomic-like $4 f^{6}$ state of $\mathrm{Sm}$ lies in the gap. In the octahedral crystal field the $d$ band is split into the $t_{2 g}$ and $e_{g}$ bands. Under pressure the crystal field split $p$ band broadens and its lower edge moves down in energy relative to the $f$ level and ultimately crosses it. The semiconductor-to-metal transition occurs at the pressure where the $f-d$ gap goes to zero. The consequent delocalisation of a $4 f$ electron yields a free $5 d$ conduction electron. This is observed by a dramatic change of colour from black to golden yellow for SmS, reduction in lattice constant and change in resistivity. In the high pressure phase of $\mathrm{SmS}$ the wave function near the narrow $f$ band is a linear combination of $f$ like and $d$ like wave functions. The occupancy of the $f$ like state decides the valence of the $\mathrm{Sm}$ ion. The Fermi level is pinned to the $f$ peak. Since the $f$ band has a non-zero width the
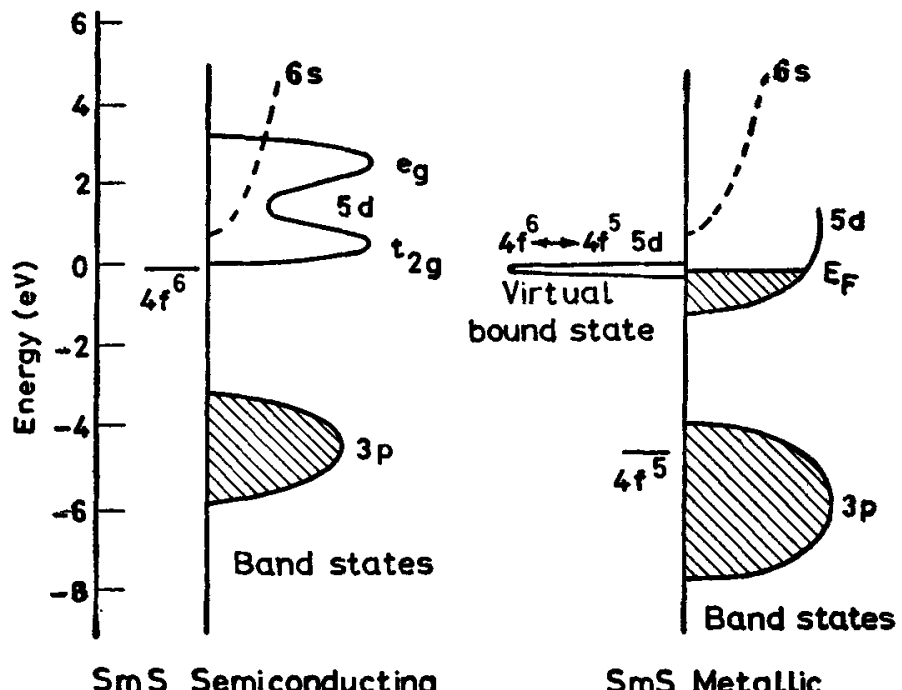

SmS Metallic

Figure 1. Schematic electronic energy level diagram of SmS (Batlogg et al 1976; Gopinathan et al 1979). 
instantaneous valence of $\mathrm{Sm}$ can vary due to fluctuation in the occupancy of the $f$ band. The fluctuation time as deduced from experiments (Shenoy 1980) is $\sim 0.6 \times 10^{-13} \mathrm{sec}$ in fair agreement with the theoretical estimate (Varma 1976).

Positron annihilation techniques are extensively applied for studies of the electronic structure and phase transitions of materials (West 1973; Dekhtyar 1974; Doyama 1979; Mijnarends 1979; Gopinathan and Viswanathan 1980). The annihilation characteristics are dependent on the nature of the electron wave function. In metals the annihilation rate is enhanced due to the polarisation of the conduction electrons by the positron. In semiconductors the presence of the band gap affects the polarisability (Brandt and Reinheimer 1970, 1971). Positron annihilation parameters change across the phase transition due to changes in the lattice constant and the band gap. Measurement of the positron lifetime gives information on the electron densities. The angular correlation and the Doppler broadening of annihilation radiations give information on the electron momentum distribution. Measurement of these characteristics in the semiconducting and metallic phases of SmS are reported here. The results are discussed in conjunction with those reported earlier (Gopinathan et al 1979; Bharathi et al 1979a).

\section{Experimental procedures and results}

Single crystals of pure $\mathrm{SmS},\left(\mathrm{Sm}_{0.8} \mathrm{Gd}_{0 \cdot 2}\right) \mathrm{S}$ and $\left(\mathrm{Sm}_{0.8} \mathrm{~Tb}_{0.2}\right) \mathrm{S}$ were used in the measurements. For positron lifetime and Doppler broadening measurements a $5 \mu \mathrm{Ci}$ carrier-free ${ }^{22} \mathrm{Na}$ source was prepared on a $1 \cdot 2 \mu$ thick pure nickel foil. The source was sandwiched between two layers of the powdered sample. The lifetime spectrometer used two fast scintillators coupled to fast photomultipliers, two constant fraction discriminators and a time-to-pulse height converter and had a resolution, $\mathrm{FWHM}=280 \mathrm{psec}$.

\subsection{Lifetime measurements in SmS under pressure}

The high pressure measurements were made in an assembly consisting of the sample enclosed in a pyrophyllite gasket of inner diameter $3 \mathrm{~mm}$ and placed between two Bridgman anvils of tungsten carbide in opposed geometry (figure 2). The required load was applied using a hydraulic press. Pressure was calibrated using the drop in resistivity in $\mathrm{SmS}$ at $6.5 \mathrm{kbar}$. The estimated uncertainty in pressure was $0.5 \mathrm{kbar}$. Measurements were made at intervals of $\sim 2 \mathrm{kbar}$ from 0 to $\sim 16$ kbar. A typical lifetime spectrum is shown in figure 3. The spectrum could be fitted to a single exponential after correcting for random coincidences which were $<0.05 \%$ at the peak. Errors due to electronic and temperature drifts were determined to be $<3 \mathrm{p} \mathrm{sec}$. Correction due to possible annihilation in the pyrophyllite gasket and the tungsten carbide anvils was found to be $<3 \mathrm{p} \mathrm{sec}$. The corrected results are given in table 1 and plotted in figure 4.

\subsection{Measurements in $\left(\mathrm{Sm}_{0 \cdot 8} G d_{0.2}\right) S$}

Lifetime and Doppler broadening measurements were made in $\left(\mathrm{Sm}_{0.8} \mathrm{Gd}_{0.2}\right) \mathrm{S}$ at various temperatures using a variable temperature liquid helium cryostat (ftgure 5). The sample assembly was attached to the copper cold finger of the 


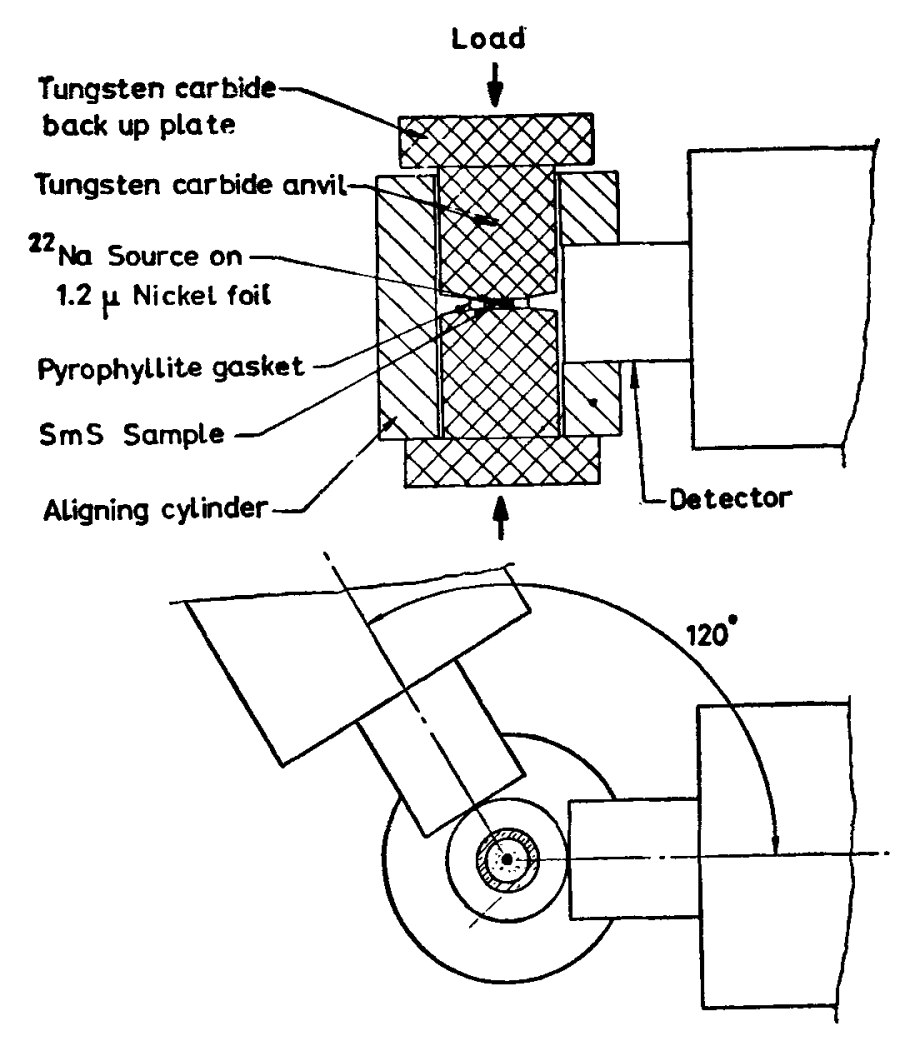

Figure 2. Schematic diagram of the high pressure assembly (Gopinathan et al 1979).

cryostat (Bharathi et al 1979b). The temperature of the sample was varied by passing a current through a heater wound around the tip of the cold-finger. The temperature was measured using a carbon or platinum resistance thermometer. At each setting the temperature was monitored continuously and was kept constant to $\pm 1 \mathrm{~K}$ for the duration of the measurement. For measurements at temperatures above $77 \mathrm{~K}$ the liquid helium in the inner cylinder of the cryostat was replaced by liquid nitrogen. The lifetime data were fitted as in the case of SmS. The results are shown in table 2 and plotted in figure 6.

Table 1. Positron lifetime in SmS vs pressure (Gopinathan et al 1979).

\begin{tabular}{llllllllll}
\hline Pressure (kbar) & 0 & $3 \cdot 0$ & $4 \cdot 4$ & $5 \cdot 9$ & $10 \cdot 3$ & $11 \cdot 8$ & $13 \cdot 3$ & $14 \cdot 8$ \\
\hline Lifetime (psec) & 343 & 341 & 345 & 342 & 323 & 320 & 326 & 324 \\
& \pm 3 & \pm 3 & \pm 3 & \pm 3 & \pm 3 & \pm 3 & \pm 3 & \pm 3 \\
\hline
\end{tabular}




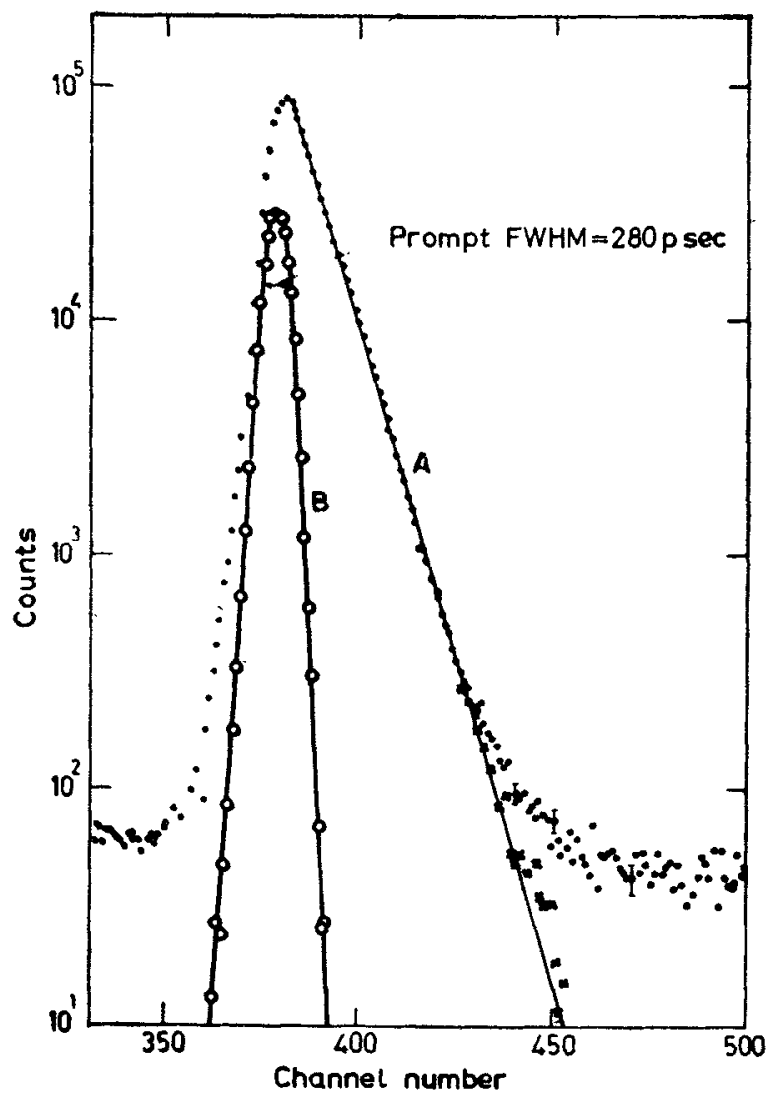

Figure 3. Typical positron lifetime spectrum of SmS (A) along with the prompt resolution spectrum using ${ }^{\circ{ }^{\circ} \mathrm{Co}}$ (B).

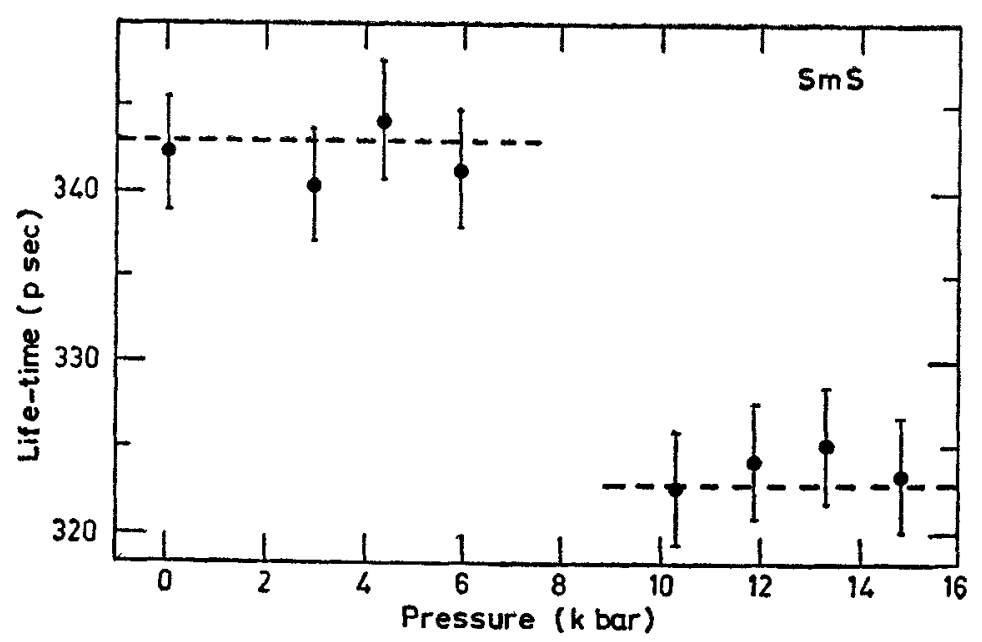

Figure 4. Positron lifetime vs pressure in SmS, 


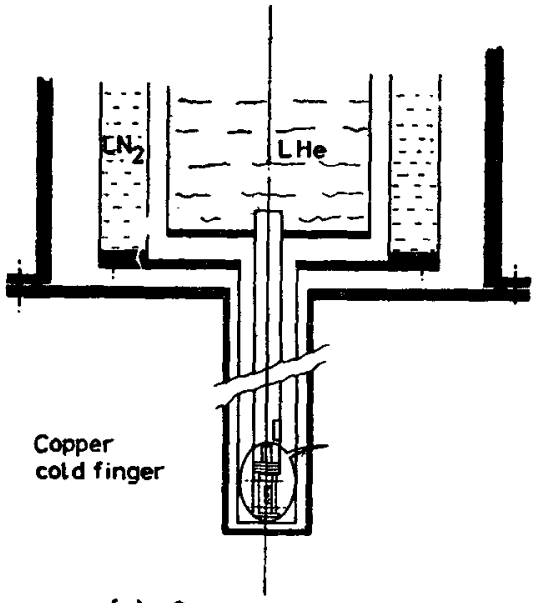

(a) Cryostat

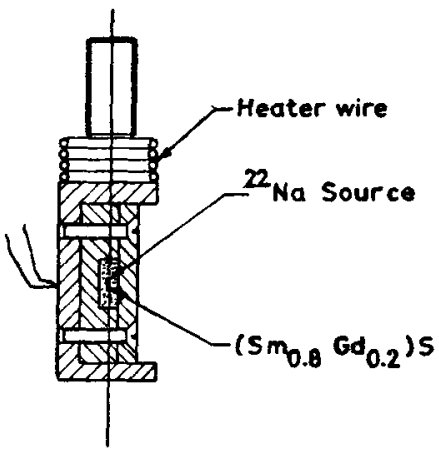

(b) Sample holder

Figure 5. Schematic diagram of the cryostat and sample assembly (Bharathi et al 1979b).

Table 2. Positron lifetime in $\left(\mathrm{Sm}_{0.8} \mathrm{Gd}_{0.8}\right) \mathrm{S}$ vs temperature (Gopirathân et al 1979)

\begin{tabular}{lrrrrrrrr}
\hline Temp. (K) & 10 & 66 & 77 & 110 & 150 & 200 & 250 & 300 \\
\hline \multirow{2}{*}{ Lifetime (psec) } & 356 & 342 & 351 & 358 & 324 & 326 & 316 & 324 \\
& \pm 5 & \pm 6 & \pm 3 & \pm 4 & \pm 5 & \pm 3 & \pm 5 & \pm 5 \\
\hline
\end{tabular}

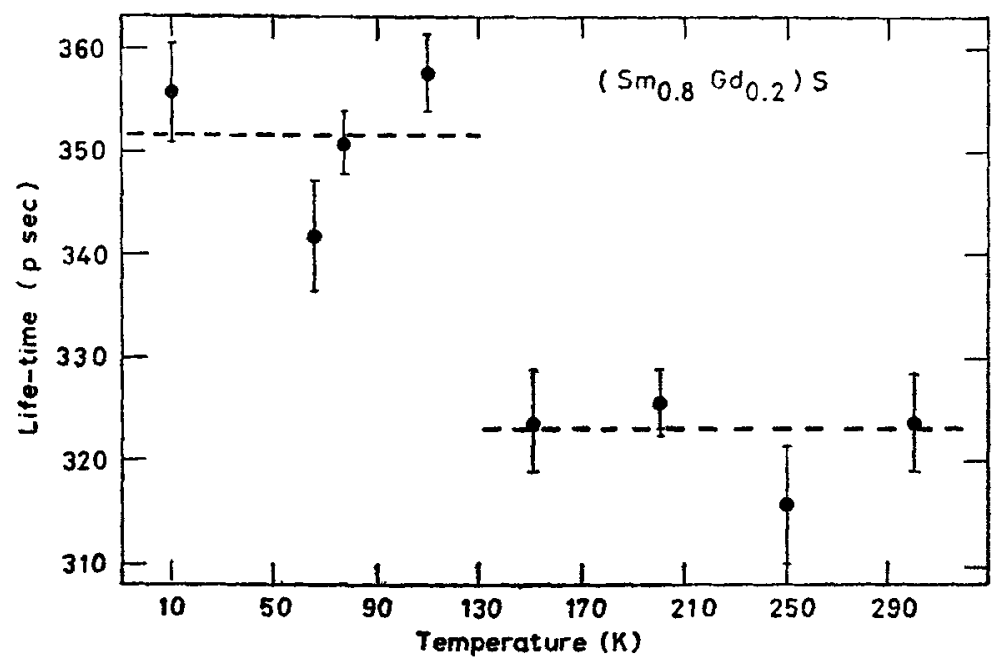

Figure 6. Positron lifetime vs temperature in $\left(\mathrm{Sm}_{0.8} \mathrm{Gd}_{0.2}\right) \mathrm{S}$. 


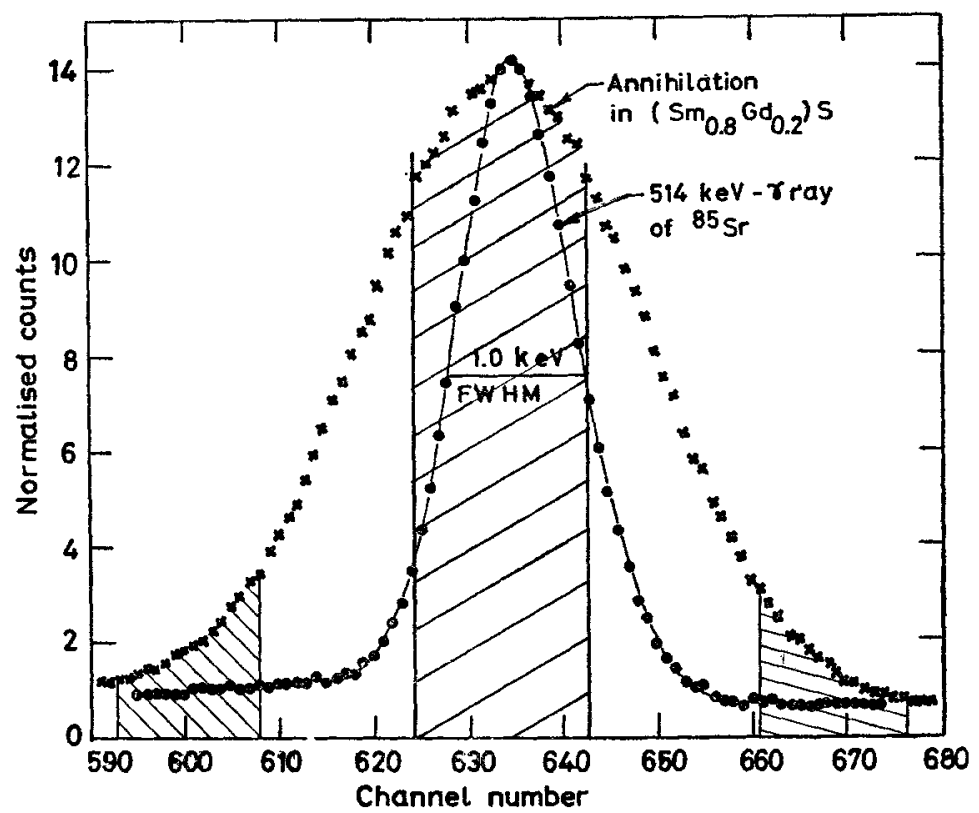

Figure 7. Typical Doppler broadened spectrum in $\left(\mathrm{Sm}_{0.8} \mathrm{Gd}_{0.2}\right) \mathrm{S}$ at $300 \mathrm{~K}$ and the resolution function of the spectrometer. The hatched regions we used for calculating the line-shape parameters.

\subsection{Doppler broadening measurements}

The Doppler broadening of positron annihilation photons in $\left(\mathrm{Sm}_{0.8} \mathrm{Gd}_{0 \cdot 2}\right) \mathrm{S}$ as a function of temperature from $\sim 300 \mathrm{~K}$ to $\sim 70 \mathrm{~K}$ was measured. The sample was made as in the case of the lifetime measurement and mounted on the coldfinger of the variable temperature cryostat. The annihilation photons were detected in a hyperpure germanium detector having an energy resolution of $1.0 \mathrm{keV}$ for the $514 \mathrm{keV}$ gamma rays of ${ }^{85} \mathrm{Sr}$. A typical Doppler broadened spectrum is shown in figure 7. The spectra were analysed in terms of the line-shape parameters $\mathrm{S}$ and $\mathrm{I}$. The $\mathrm{S}$ parameter is defined as the ratio of the total counts under the central 21 channels to the sum of the counts under 15 channels on either wing

Table 3. Line-shape parameters in $\left(\mathrm{Sm}_{0.8} \mathrm{Gd}_{0.2}\right) \mathrm{S}$ vs temperature

\begin{tabular}{|c|c|c|c|c|c|c|c|c|}
\hline $\begin{array}{c}\text { Temp. } \\
\text { (K) }\end{array}$ & 71 & 88 & 105 & 114 & 140 & 179 & 243 & 300 \\
\hline \multirow[t]{2}{*}{$\mathbf{s}$} & $6 \cdot 04$ & $6 \cdot 08$ & 6.06 & $6 \cdot 07$ & 5.99 & $5 \cdot 80$ & 5.90 & $5 \cdot 69$ \\
\hline & $\pm \cdot 03$ & $\pm \cdot 02$ & $\pm \cdot 02$ & $\pm \cdot 02$ & $\pm \cdot 02$ & $\pm \cdot 03$ & $\pm \cdot 03$ & $\pm \cdot 03$ \\
\hline \multirow[t]{2}{*}{$\mathbf{I}$} & 0.4877 & 0.4877 & 0.4884 & $0 \cdot 4884$ & 0.4866 & 0.4827 & 0.4843 & 0.4800 \\
\hline & $\pm \cdot 0003$ & $\pm \cdot 0002$ & $\pm \cdot 0002$ & $\pm \cdot 0.002$ & $\pm \cdot 0002$ & $\pm \cdot 0004$ & $\pm \cdot 0003$ & $\pm \cdot c 003$ \\
\hline
\end{tabular}


of the photopeak of the $511 \mathrm{keV}$ annihilation radiation (see figure 7). The parameter $I$ is defined as the ratio of the area under the central 21 channels to the total area under the photopeak. The values of the $S$ and I parameters for $\left(\mathbf{S m}_{0.8} \mathbf{G d}_{0.2}\right) S$ at various temperatures are shown in table 3 and plotted in figure 8.

\subsection{Angular correlation measurements}

The angular correlation of positron annihilation photons in the semiconducying phase of $\mathrm{SmS}$ and in the metallic phase of $\left(\mathrm{Sm}_{0} \cdot \mathrm{Tb}_{0 \cdot 2}\right) \mathrm{S}$ has been measured. The apparatus used for this measurement (figure 9) has the long-slit geometry (West 1973; Dekhtyar 1974; Hautojarvi and Vehanen 1979; Mijnarends 1979) and hence measures the $z$-component of the momentum of the annihilating electronpositron pair. It consists of a shielded sample and source chamber, a fixed and a movable detector, each placed on 3 metre long arms on either side of the sample chamber, a set of coarse and fine collimators and an electronic set-up for coincidence measurements. The detectors consist of $30 \mathrm{~cm}$ long $\times 5 \mathrm{~cm}$, diameter NaI(T1) scintillators coupled to RCA 8575 photomultipliers. The fine slits were adjusted to get an angular resolution of $1 \mathrm{mrad}$ in the present measurements. The detectors were connected to a fast-slow coincidence circuit having a resolving time of $60 n$ sec. A $10 \mathrm{mCi}{ }^{22} \mathrm{Na}$ source was placed in the source chamber shielded from the detectors. The sample was suspended at the centre of the chamber a few $\mathrm{mm}$ above the source. The windows on either side of the chamber allowed only the annihilation photons emerging out of the sample to come out of the chamber and be counted by the detectors. The coincidence counts were recorded as a function of the angular position, $\theta_{z}$ of the movable arm. Typical angular correlation curves measured by the apparatus are shown in figure 10.

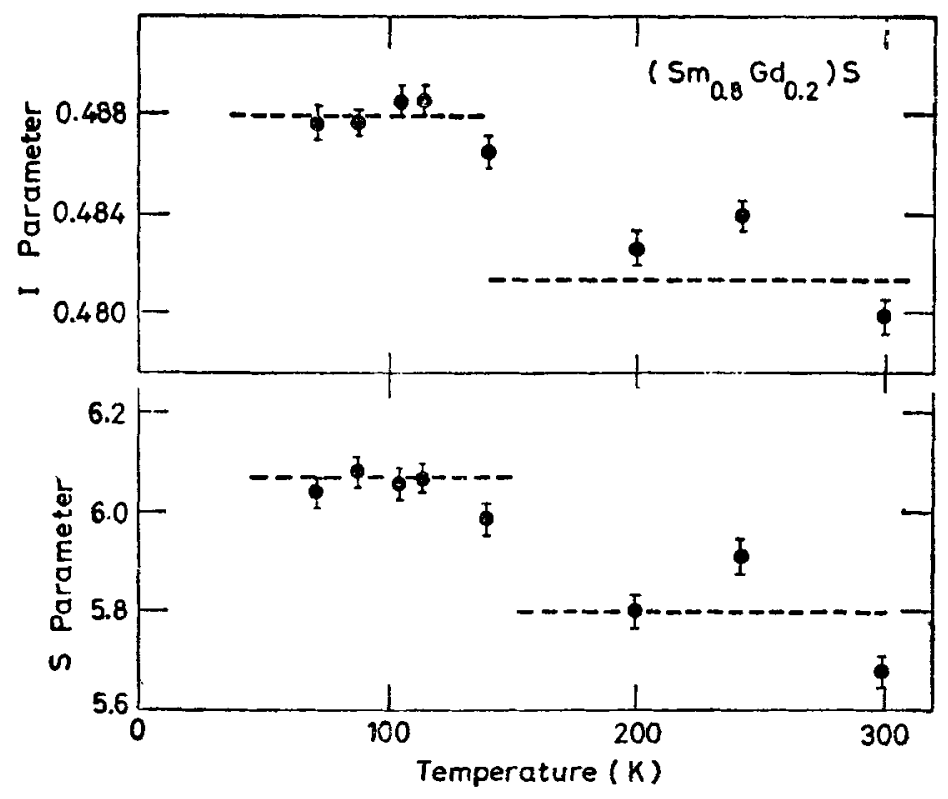

Figure 8. Line-shape parameters $S$ and $I$ in $\left(\mathrm{Sm}_{0.8} \mathrm{Gd}_{0.2}\right) S$ vs temperature. 


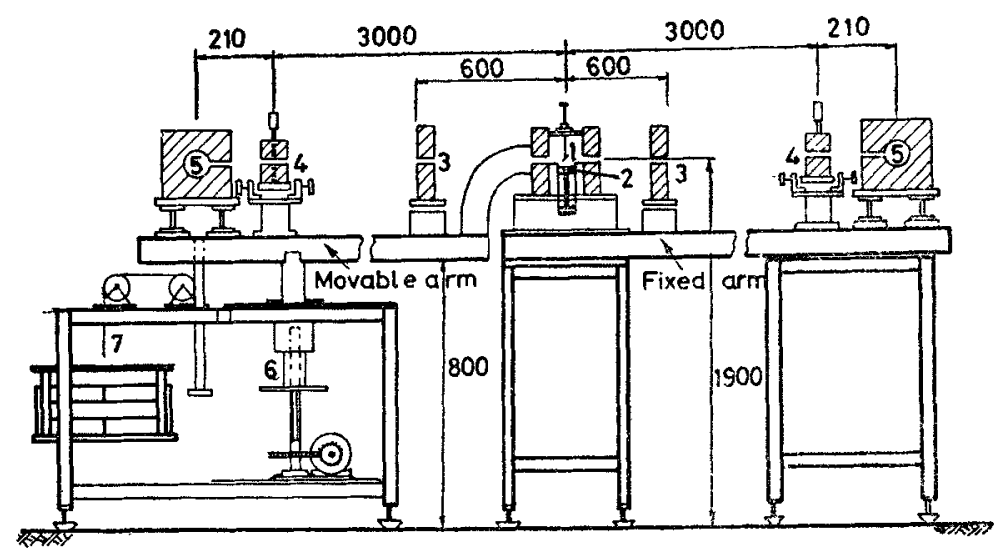

Figure 9. Schematic diagram of the angular correlation set-up : 1-sample, 2source, 3-coarse collimators, 4-fine collimators, 5-detectors, 6-drive shaft and screw, 7-counter-weight. The distances are shown in mm.

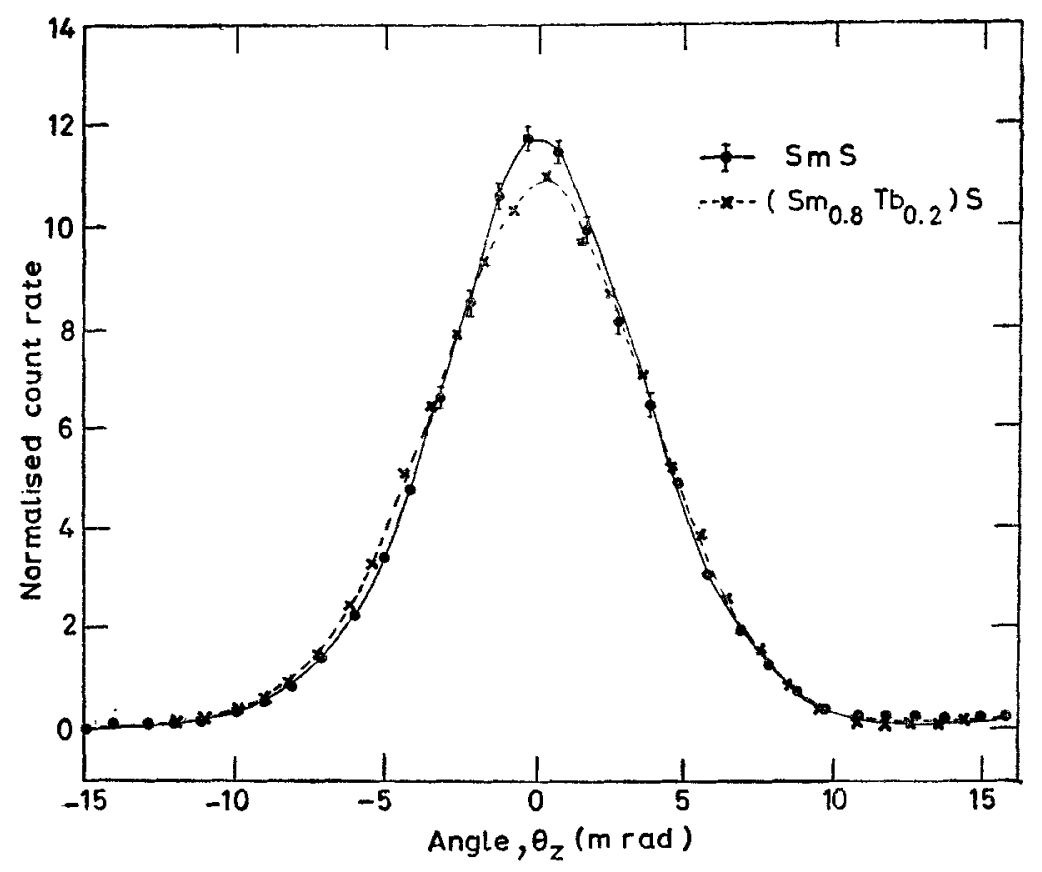

Figure 10. Typical angular correlation curves in $\mathrm{SmS}$ and $\left(\mathrm{Sm}_{0.8} \mathrm{~Tb}_{0.2}\right) \mathrm{S}$.

\section{Discussion}

The positron lifetime in SmS shows an abrupt decrease at $6.5 \mathrm{kbar}$ due to the transformation from the semiconducting phase to the metallic phase (figure 4). Similar discontinuities in the lifetime (figure 6) and Doppler line-shape parameters (figure 8) are observed in $\left(\mathrm{Sm}_{0.8} \mathrm{Gd}_{0.2}\right) \mathrm{S}$. The Doppler broadening is larger in the metallic than in the non-metallic phase. The angular correlation profile in metallic $\left(\mathrm{Sm}_{0.8} \mathrm{~Tb}_{0.2}\right) \mathrm{S}$ is found to be broader (figure 10) as compared to that in the semiconducting phase of SmS, 
The samarium chalcogenides are ionic crystals. Hence positrons predominantly annihilate with the $3 p^{6}$ valence electrons of the sulphur anions in the non-metallic phase. However in the metallic phase there are two differences, viz., (i) an additional contribution to the annihilation rate from the electrons in the $5 d=6 s$ conduction band (see figure 1) and (ii) the smaller lattice constant of the metallic phase. By taking these effects into account the change in the lifetime in the semiconducting and metallic phases of SmS has been explained (Gopinathan et al 1979).

The reduced lattice constant and the $5 d-6 s$ conduction electron contribution in the metallic phase of $\left(\mathrm{Sm}_{0 \cdot 8} \mathrm{Gd}_{0 \cdot 2}\right) \mathrm{S}$ and $\left(\mathrm{Sm}_{0.8} \mathrm{~Tb}_{0.2}\right) \mathrm{S}$ increase the Fermi momentum and broaden the electron momentum distribution as compared to that in the semiconducting phase. This explains the reduction in the Doppler line-shape parameters and the broadening of the angular correlation curve in the metallic phase relative to that in the non-metallic phase.

\section{Acknowledgements}

The authors thank Dr G Venkataraman for his interest in this work and Dr A Jayaraman and Dr R Suryanarayanan for providing the samples. They are grateful to Shri S K Sarkar for his assistance in instrumentation.

\section{References}

Batlogg B, Kaldis E, Schlegel A and Wachter P 1976 Phys. Rev. B14 5503

Bharathi A, Gopinathan K P, Sarkar S K, Sundar C S and Viswanathan B 1979a Nucl. Phys. Solid State Phys. (India) $\mathbf{C 2 2}$ (in print)

Bharathi A, Gopinathan K P, Sundar C S and Viswanathan B 19796 Pramāna 13625

Brandt W and Reinheimer J 1970 Phys. Rev. B2 3104

Brandt W and Reinheimer J 1971 Phys. Lett. A35 109

Campagna M, Bucher E, Wertheim G K and Longinotti L D 1974 Phys. Rev. Lett. 33165

Coey J M D, Ghatak S K, Avignon M and Holtzberg F 1976 Phys. Rev. B14 3744

Dekhtyar I Ya 1974 Phys. Rep. 9243

Doyama M 1979 Proc. Fifth Int. Conf. Positron Annihilation, Lake Yamanaka, Japan, (eds) R R Hasiguti and K Fujiwara (Sendai : The Japan Institute of Metals) p. 13

Gopinathan K P, Sundar C S and Viswanathan B 1979 Solid State Commun. 32369 ; Proc. Fifth Int. Conf. Positron Annihilation, Lake Yamanaka, Japan, (eds.) R R Hasiguti and K Fujiwara (Sendai : The Japan Institute of Metals) p. 487

Gopinathan K P and Viswanathan B 1980 in Positron annihilation (eds) P C Jain and R M Singru (New Delhi : South Asian Publishers) p. 27

Hautojarvi P and Vehanen A 1979 in Positrons in solids (ed.) P Hautojarvi (Berlin : SpringerVerlag) p. 1

Jayaraman A, Narayanamurti V, Bucher E and Maines R G 1970 Phys. Rev. Lett. 25 368, 1430 Jayaraman A, Bucher E, Dernier P D and Longinotti L D 1973 Phys. Rev. Lett. 31700

Jayaraman A, Dernier P D and Longinotti L D 1975a Phys. Rev. B11 2783

Jayaraman A, Dernier P D and Longinotti L D 1975b High Temp. High Pressures 71

Mijnarends P E 1979 in Positrons in solids (ed.) P Hautojarvi (Berlin : Springer-Verlag) p. 25

Pollak R A, Holtzberg F, Freeouf J L and Eastman D 1974 Phys. Rev. Lett. 33820

Shenoy G K 1980 in Science and technology of rare earth materials, eds E C Subba Rao and W E Wallace (New York: Academic Press) (in print)

Suryanarayanan R 1978 Phys. Stat. Sol. $\mathrm{B85} 9$

Varma C M 1976 Rev. Mod. Phys. 48219

West R N 1973 Adv. Phys. 22263 